\title{
Enhancement of satellite image compression using a hybrid (DWT-DCT) algorithm
}

\begin{abstract}
Discrete Cosine Transform (DCT) and Discrete Wavelet Transform (DWT) image compression techniques have been utilized in most of the earth observation satellites launched during the last few decades. However, these techniques have some issues that should be addressed. The DWT method has proven to be more efficient than DCT for several reasons. Nevertheless, the DCT can be exploited to improve the high-resolution satellite image compression when combined with the DWT technique. Hence, a proposed hybrid (DWT-DCT) method was developed and implemented in the current work, simulating an image compression system on-board on a small remote sensing satellite, with the aim of achieving a higher compression ratio to decrease the onboard data storage and the downlink bandwidth, while avoiding further complex levels of DWT. This method also succeeded in maintaining the reconstructed satellite image quality through replacing the standard forward DWT thresholding and quantization processes with an alternative process that employed the zero-padding technique, which also helped to reduce the processing time of DWT compression. The DCT, DWT and the proposed hybrid methods were implemented individually, for comparison, on three LANDSAT 8 images, using the MATLAB software package. A comparison was also made between the proposed method and three other previously published hybrid methods. The evaluation of all the objective and subjective results indicated the feasibility of using the proposed hybrid (DWT-DCT) method to enhance the image compression process on-board satellites.e nutritional and antioxidant properties of underutilized BRF as a food ingredient.
\end{abstract}

Keyword: Image compression; Discrete cosine transform; Discrete wavelet transform; Satellite images; Remote sensing 